\title{
THE AVERAGE METHOD IS MUCH BETTER THAN AVERAGE
}

\author{
Lívia Boda AND IsTVÁN FARAGó \\ Department of Differential Equations, Budapest University of Technology and Economics, \\ Müegytem rkp. 3., H-1111 Budapest, Hungary \\ faragois@gmail.com, bodalivi@math.bme.hu \\ TAMÁS KALMÁR-NAGY \\ Department of Fluid Mechanics, Budapest University of Technology and Economics, \\ Müegytem rkp. 3., H-1111 Budapest, Hungary \\ kalmarnagy@ara.bme.hu
}

[Received: October 14, 2020; Accepted: November 21, 2020]

Dedicated to Professor Barna Szabó on the occasion of his eighty-fifth birthday

\begin{abstract}
Operator splitting is a powerful method for the numerical investigation of complex time-dependent models, where the stationary (elliptic) part consists of a sum of several structurally simpler sub-operators. As an alternative to the classical splitting methods, a new splitting scheme is proposed here, the Average Method with sequential splitting. In this method, a decomposition of the original problem is sought in terms of commuting matrices. Wedemonstrate that third-order accuracy can be achieved with the Average Method. The computational performance of the method is investigated, yielding run times 1-2 orders of magnitude faster than traditional methods.
\end{abstract}

Mathematical Subject Classification: 65L05, 76G25

Keywords: Operator splitting, Cauchy problem, numerical solution

\section{INTRODUCTION}

Operator splitting entails the decomposition (splitting) of the spatial differential operator of the problem into a sum of different sub-operators having simpler forms. Operator splitting methods are commonly used in many applications. The decomposition can be motivated mathematically (equations of different types, elliptic, parabolic, etc.) or by the presence of subsystems described by different areas of physics (coupled flow-structure or thermo-mechanical problems, for example).

McLahlan and Quispel [1] survey splitting methods for the numerical integration of ODE's. A nice exposition of splitting methods can be found in 2. Different communities use different names for the same concept. Operator splitting is also called staggered methods (schemes), decomposition, co-simulation, etc. Gu and Asada [3] discuss the concept of co-simulation, which refers to simultaneous numerical solution 
(discretization) of multiple interacting subsystem. A simple mathematical model for co-simulation is proposed in [4], together with the study of the resulting stability charts. Csomós and Nickel introduce splitting methods for delay equations in an abstract setting 5 and prove the convergence of the method.

The structure of this paper is the following. In Section 2 we discuss two basic splitting methods: sequential and Strang-Marchuk splitting. Then we introduce the Average Method in Section 3. In the same section we discuss the possible reduction of the terms needed for the Average Method by using a matrix decomposition of pairwise commuting matrices. In Section 4 we state a condition that makes the Average Method with sequential splitting third-order in accuracy. Here we also show that third-order accuracy cannot be achieved when basing the Average Method on Strang-Marchuk splitting. In Section 5 an example problem is given, motivated by an aerodynamic model. In Section 6 results of eight runtimes of the various methods. Section 7 summarizes the findings of this work.

\section{Sequential and Strang-Marchuk splitting}

This section is based on 2]. We consider the following Cauchy problem in $\mathbb{R}^{m}$

$$
\left\{\begin{array}{l}
\dot{y}(t)=A y(t)=\sum_{i=1}^{d} A_{i} y(t) \quad t \in[0, T) \\
y(0)=y_{0},
\end{array}\right.
$$

where $y:[0, T] \rightarrow \mathbb{R}^{m}$ is the unknown function, $y_{0} \in \mathbb{R}^{m}$ is the given initial vector, $A_{i} \in \mathbb{R}^{m \times m}(i=1, \ldots, d)$ are matrices.

The exact solution of the Cauchy problem (1) can be written directly as

$$
y(t)=\exp (t A) y(0) \text {. }
$$

Our aim is to approximate the exact solution numerically on the grid

$$
\omega_{h}=\left\{t_{n}=n \cdot h, h=\frac{T}{N}, n=0,1, \ldots, N\right\} .
$$

In sequential splitting we decompose the original problem (1) into $d$ sub-problems $(i=1,2, \ldots, d)$

$$
\left\{\begin{array}{l}
\dot{y}_{i}^{n}(t)=A_{i} y_{i}^{n}(t), \\
y_{i}^{n}((n-1) h)=y_{i-1}^{n}(n h)
\end{array} \quad t \in((n-1) h, n h]\right.
$$

The solution is

$$
y_{\text {seq }}^{N}(n h)=y_{d}^{n}(n h)
$$

where $y_{0}^{n}(n h)=y_{\text {seq }}^{N}((n-1) h)$, and $y_{\text {seq }}^{N}(0)=y(0)=y_{0}$.

Sequential splitting is a first-order method.

The main difference between sequential and Strang-Marchuk splitting is that the latter computes the values in the midpoints of the subintervals. First

$$
\left\{\begin{array}{l}
\dot{y}_{i}^{n}(t)=A_{i} y_{i}^{n}(t), \\
y_{i}^{n}((n-1) h)=y_{i-1}^{n}\left(\left(n-\frac{1}{2}\right) h\right)
\end{array} \quad t \in\left((n-1) h,\left(n-\frac{1}{2}\right) h\right]\right.
$$


is computed for $i=1,2, \ldots, d-1$. For $i=d$ the slightly different

$$
\left\{\begin{array}{l}
\dot{y}_{d}^{n}(t)=A_{d} y_{d}^{n}(t), \\
y_{d}^{n}((n-1) h)=y_{d-1}^{n}\left(\left(n-\frac{1}{2}\right) h\right)
\end{array} \quad t \in((n-1) h, n h],\right.
$$

is evaluated. For $i=d+1, \ldots, 2 d-1$ the following formula is used

$$
\left\{\begin{array}{l}
\dot{y}_{i}^{n}(t)=A_{i} y_{i}^{n}(t), \\
y_{i}^{n}\left(\left(n-\frac{1}{2}\right) h\right)=y_{i-1}^{n}(n h) .
\end{array} \quad t \in\left(\left(n-\frac{1}{2}\right) h, n h\right],\right.
$$

The solutions are given by

$$
y_{S M}^{N}(n h)=y_{2 d-1}^{n}(n h),
$$

where $y_{0}^{n}\left(\left(n-\frac{1}{2}\right) h\right)=y_{S M}^{N}((n-1) h)$ and $y_{S M}^{N}(0)=y(0)=y_{0}$.

The Strang-Marchuk method is a second-order method.

\section{The Average Method}

A new method is introduced here (referred to as the Average Method) based on the following idea: dividing the Cauchy problem (1) into $d$ subproblems, using sequential splitting in all possible sequences, calculating the numerical solutions and then taking their arithmetic mean and letting it be the numerical solution in $\omega_{h}$.

Let $\mathcal{P}^{n}$ denote the set for the permutations of the indices $\{1,2, \ldots, n\}$. For $p=$ $\left\{p_{1}, p_{2}, \ldots, p_{n}\right\} \in \mathcal{P}^{n}$ we introduce the notation

$$
\exp \left\{p_{1}, p_{2}, \ldots, p_{n}\right\}=\exp \left(h A_{p_{1}}\right) \exp \left(h A_{p_{2}}\right) \cdot \ldots \cdot \exp \left(h A_{p_{n}}\right) .
$$

Solving the Cauchy-problem (1) using sequential splitting for all possible permutations and then averaging the resulting numerical solutions yields a second-order method, i.e.

\section{Statement 1.}

$$
\exp \left(h\left(A_{1}+\ldots+A_{d}\right)\right)=\frac{1}{d !} \sum_{p \in \mathcal{P}^{d}} \exp \left\{p_{1}, p_{2}, \ldots, p_{n}\right\}+\mathcal{O}\left(h^{3}\right) .
$$

See Appendix 1 for the proof.

Since $\mathcal{P}^{d}$ has $d$ ! elements, we examine conditions that reduce the complexity of the Average Method. Consider the case of $d=3$, i.e. $A=A_{1}+A_{2}+A_{3}$. By Statement 1 we have

$$
\exp \left(h\left(A_{1}+\ldots+A_{d}\right)\right)=\frac{1}{3 !} \sum_{p \in \mathcal{P}^{3}} \exp \left\{p_{1}, p_{2}, p_{3}\right\}
$$

with $3 !=6$ terms on the right-hand side. We utilize the usual definition of the commutator $[A, B]=A B-B A$. If, for example, $A_{1}$ and $A_{3}$ commute, i.e. $\left[A_{1}, A_{3}\right]=\mathbf{0}$, then we have

$$
\begin{aligned}
& \exp \left\{p_{2}, p_{1}, p_{3}\right\}=\exp \left\{p_{2}, p_{3}, p_{1}\right\} \\
& \exp \left\{p_{1}, p_{3}, p_{2}\right\}=\exp \left\{p_{3}, p_{1}, p_{2}\right\}
\end{aligned}
$$

and 


$$
\begin{aligned}
& \sum_{p \in \mathcal{P}^{3}} \exp \left\{p_{1}, p_{2}, p_{3}\right\}= \\
& \quad=\exp \left\{p_{1}, p_{2}, p_{3}\right\}+\exp \left\{p_{3}, p_{2}, p_{1}\right\}+2 \exp \left\{p_{2}, p_{1}, p_{3}\right\}+2 \exp \left\{p_{1}, p_{3}, p_{2}\right\}
\end{aligned}
$$

where the number of terms was reduced from six to four.

Let us now consider the general case. Let $A=A_{1}+A_{2}+\ldots+A_{d}$, and suppose that $\exists$ $i, j \in \mathbb{N}, i \neq j$ such that $\left[A_{i}, A_{j}\right]=0$. Then instead of all the $d$ ! permutations, we have $d !-(d-1) !=(d-1)(d-1)$ ! elements. If the decomposition includes more commuting pairs of matrices, the reduction might be more significant. An interesting question is the decomposition of a given matrix into pairwise commuting matrices. Even the study of the pairs of $n \times n$ commuting matrices $A$ and $B$ yields non-trivial results (i.e. Schur's theorem, Gerstenhaber's theorem, see, for example, Section 5 of [6]). The so-called commuting variety generated by the $n^{2}$ equations $(A B)_{i j}-(B A)_{i j}=0$ can be investigated with the tools of algebraic geometry or linear algebraic conditions can be sought for commutativity. For $k$ matrices one deals with commutative $k$-generated subalgebras, where even the best upper bound for their dimension is an open problem.

\section{Making the Average Method third-order}

Now we consider if third-order accuracy can be achieved with the Average Method based on sequential splitting. Assume that we have the Cauchy problem (1), with $d=2$. We then have the following

Statement 2. If and only if $A=A_{1}+A_{2}$, and $A_{1}$ and $A_{2}$ satisfy the condition

$$
\begin{aligned}
& {\left[A_{1},\left[A_{1}, A_{2}\right]\right]=\left[A_{2},\left[A_{1}, A_{2}\right]\right] \text { then }} \\
& \quad \exp \left(h\left(A_{1}+A_{2}\right)\right)=\frac{\exp \left(h A_{1}\right) \exp \left(h A_{2}\right)+\exp \left(h A_{2}\right) \exp \left(h A_{1}\right)}{2}+\mathcal{O}\left(h^{4}\right) .
\end{aligned}
$$

See Appendix 2 for the proof.

Now we consider the Strang-Marchuk splitting as the base method of average splitting. Can third-order accuracy be obtained by the Average Method?

The decomposition $A=A_{1}+A_{2}$ with weights $\alpha$ and $\beta(\alpha, \beta \neq 0$ and $\alpha+\beta \neq 0)$ has to satisfy

$$
\begin{aligned}
& \exp \left(h\left(A_{1}+A_{2}\right)\right)= \\
& =\frac{\alpha\left(\exp \left(\frac{h A_{1}}{2}\right) \exp \left(h A_{2}\right) \exp \left(\frac{h A_{1}}{2}\right)\right)+\beta\left(\exp \left(\frac{h A_{2}}{2}\right) \exp \left(h A_{1}\right) \exp \left(\frac{h A_{2}}{2}\right)\right)}{\alpha+\beta}+ \\
& +O\left(h^{4}\right)
\end{aligned}
$$

The right-hand side of equation $(16)$ can be written as 


$$
\begin{aligned}
& \frac{(\alpha+\beta) I+h(\alpha+\beta)\left(A_{1}+A_{2}\right)+\frac{h^{2}}{2 !}(\alpha+\beta)\left(A_{1}^{2}+A_{2}^{2}+A_{1} A_{2}+A_{2} A_{1}\right)}{\alpha+\beta}+ \\
& +\frac{h^{3}}{3 !}\left(\frac{(\alpha+\beta)\left(A_{1}^{3}+A_{2}^{3}\right)}{\alpha+\beta}+\frac{\left(\frac{3}{4} \alpha+\frac{3}{2} \beta\right) A_{1}^{2} A_{2}+\left(\frac{3}{4} \alpha+\frac{3}{2} \beta\right) A_{2} A_{1}^{2}+\left(\frac{3}{2} \alpha+\frac{3}{4} \beta\right) A_{2}^{2} A_{1}}{\alpha+\beta}+\right. \\
& \left.+\frac{\left(\frac{3}{2} \alpha+\frac{3}{4} \beta\right) A_{1} A_{2}^{2}+\frac{3}{2} \alpha A_{1} A_{2} A_{1}+\frac{3}{2} \beta A_{2} A_{1} A_{2}}{\alpha+\beta}\right)+O\left(h^{4}\right)= \\
& =I+h\left(A_{1}+A_{2}\right)+\frac{h^{2}}{2 !}\left(A_{1}^{2}+A_{2}^{2}+A_{1} A_{2}+A_{2} A_{1}\right)+\frac{h^{3}}{3 !}\left(\left(A_{1}^{3}+A_{2}^{3}\right)+\right. \\
& +\frac{\left(\frac{3}{4} \alpha+\frac{3}{2} \beta\right) A_{1}^{2} A_{2}+\left(\frac{3}{4} \alpha+\frac{3}{2} \beta\right) A_{2} A_{1}^{2}+\left(\frac{3}{2} \alpha+\frac{3}{4} \beta\right) A_{2}^{2} A_{1}}{\alpha+\beta}+ \\
& \left.+\frac{\left(\frac{3}{2} \alpha+\frac{3}{4} \beta\right) A_{1} A_{2}^{2}+\frac{3}{2} \alpha A_{1} A_{2} A_{1}+\frac{3}{2} \beta A_{2} A_{1} A_{2}}{\alpha+\beta}\right)+O\left(h^{4}\right) .
\end{aligned}
$$

The left-hand side of Equation 16 is

$$
\begin{aligned}
& \exp \left(h\left(A_{1}+A_{2}\right)\right)=I+h\left(A_{1}+A_{2}\right)+\frac{h^{2}}{2 !}\left(A_{1}+A_{2}\right)^{2}+\frac{h^{3}}{3 !}\left(A_{1}+A_{2}\right)^{3}+\mathcal{O}\left(h^{4}\right)= \\
& =I+h\left(A_{1}+A_{2}\right)+\frac{h^{2}}{2}\left(A_{1}^{2}+A_{2}^{2}+A_{1} A_{2}+A_{2} A_{1}\right)+ \\
& +\frac{h^{3}}{6}\left(A_{1}^{3}+A_{2}^{3}+A_{1} A_{2}^{2}+A_{1}^{2} A_{2}+A_{2} A_{1}^{2}+A_{2}^{2} A_{1}+A_{1} A_{2} A_{1}+A_{2} A_{1} A_{2}\right)+\mathcal{O}\left(h^{4}\right) .
\end{aligned}
$$

The equality $(16)$ is only true under the conditions

$$
\begin{gathered}
\frac{\frac{3}{4} \alpha+\frac{3}{2} \beta}{\alpha+\beta}=1, \\
\frac{\frac{3}{2} \alpha+\frac{3}{4} \beta}{\alpha+\beta}=1, \\
\frac{\frac{3}{2} \alpha}{\alpha+\beta}=1, \\
\frac{\frac{3}{2} \beta}{\alpha+\beta}=1 .
\end{gathered}
$$

Equations (19) and 21) give the condition $\alpha=2 \beta$, while Equation (22) yields the condition $\beta=2 \alpha$. This implies $\alpha=\beta=0$, which contradicts our assumption.

Third-order accuracy thus cannot be achieved with the Average Method based on Strang-Marchuk splitting. 


\section{EXAmple ApPlication}

As seen in Section 3, the number of terms needed for the Average Method can be reduced by decomposing the underlying matrix into a set of matrices that have commuting elements.

We now investigate the efficacy of the three splitting methods discussed above on a physical problem. The model was chosen because of the structure of the matrices involved, i.e. sparse matrices whose decomposition into a partially commuting set was easy.

A piecewise-linear model of flutter was investigated in 7$]$ and 8 . The affine model equations contain the three system matrices $(k=0,1,2)$

$$
A_{k}=\left(\begin{array}{cccc}
0 & 1 & 0 & 0 \\
-1 & -\left(p_{1}+p_{2} \mu c_{k}\right) & -\mu^{2} c_{k} p_{2} & 0 \\
0 & 0 & 0 & 1 \\
0 & c_{k} \mu & -\left(p_{4}-c_{k} \mu^{2}\right) & -p_{3}
\end{array}\right)
$$

with the model parameters given in Table 1 (see $[7]$ ) and $\mu \in(0, \infty)$ represents the nondimensional wind speed.

Table 1. Parameters of the model

\begin{tabular}{|l|c|c|c|c|c|c|c|c|c|}
\hline Parameter & $c_{0}$ & $c_{1}$ & $c_{2}$ & $d_{1}$ & $d_{2}$ & $p_{1}$ & $p_{2}$ & $p_{3}$ & $p_{4}$ \\
\hline Value & 5.932 & -6.846 & 2.662 & 2.56 & -0.2515 & 0.1485 & 0.0147 & 0.0540 & 0.2748 \\
\hline
\end{tabular}

Motivated by this model, we consider the following 4-dimensional Cauchy problem

$$
\left\{\begin{array}{l}
\dot{\mathbf{x}}(t)=A_{k} \mathbf{x}(t), \\
\mathbf{x}(0)=\mathbf{x}_{0} .
\end{array}\right.
$$

5.1. Decompositions of matrix $A_{k}$. All three $A_{k}$ matrices have the same structure, thus we can discuss the symbolic decompositions of $A_{k}$.

First, we analyze the decomposition

$$
A_{k}=A_{k_{(1)}}+A_{k_{(2)}}
$$

where

$$
A_{k_{(1)}}=\left(\begin{array}{cccc}
0 & 1 & 0 & 0 \\
0 & -\left(p_{1}+p_{2} \mu c_{k}\right) & -\mu^{2} c_{k} p_{2} & 0 \\
0 & 0 & 0 & 1 \\
0 & 0 & 0 & -p_{3}
\end{array}\right), \quad A_{k_{(2)}}=\left(\begin{array}{cccc}
0 & 0 & 0 & 0 \\
-1 & 0 & 0 & 0 \\
0 & 0 & 0 & 0 \\
0 & c_{k} \mu-\left(p_{4}-c_{k} \mu^{2}\right) & 0
\end{array}\right) .
$$

Clearly, $A_{k_{(1)}}$ is an upper triangular matrix. On the other hand, $A_{k_{(2)}}$ is a strictly lower triangular matrix and hence it is nilpotent. Therefore $\left(A_{k_{(2)}}\right)^{m}=\mathbf{0}$ for $m>2$. This means that the exponential of the matrix $A_{k_{(2)}}$ can be computed exactly. Thus, when we realize the splitting methods, the solution of the subproblem with this matrix can be calculated exactly. We can also define a decomposition in which the exponential of each matrix can be calculated exactly. For example, the decomposition

$$
A_{k}=A_{k_{(1)}}+A_{k_{(2)}}+A_{k_{(3)}},
$$


with

$$
\begin{gathered}
A_{k_{(1)}}=\left(\begin{array}{cccc}
0 & 1 & 0 & 0 \\
0 & 0 & -\mu^{2} c_{k} p_{2} & 0 \\
0 & 0 & 0 & 1 \\
0 & 0 & 0 & 0
\end{array}\right), \quad A_{k_{(2)}}=\left(\begin{array}{ccccc}
0 & 0 & 0 & 0 \\
0 & -\left(p_{1}+p_{2} \mu c_{k}\right) & 0 & 0 \\
0 & 0 & 0 & 0 \\
0 & 0 & 0 & -p_{3}
\end{array}\right), \\
A_{k_{(3)}}=\left(\begin{array}{cccc}
0 & 0 & 0 & 0 \\
-1 & 0 & 0 & 0 \\
0 & 0 & 0 & 0 \\
0 & c_{k} \mu & -\left(p_{4}-c_{k} \mu^{2}\right) & 0
\end{array}\right)
\end{gathered}
$$

has this property because it is the sum of two nilpotent matrices and a diagonal matrix.

Now we define three decompositions which consist of commuting matrices. First, we consider the decomposition:

$$
A_{k}=A_{k_{(1)}}+A_{k_{(2)}}+A_{k_{(3)}}
$$

where

$$
\begin{gathered}
A_{k_{(1)}}=\left(\begin{array}{cccc}
0 & 1 & 0 & 0 \\
-1 & -\left(p_{1}+p_{2} \mu c_{k}\right) & 0 & 0 \\
0 & 0 & 0 & 0 \\
0 & 0 & 0 & 0
\end{array}\right), \quad A_{k_{(2)}}=\left(\begin{array}{cccc}
0 & 0 & 0 & 0 \\
0 & 0 & 0 & 0 \\
0 & 0 & 0 & 1 \\
0 & 0 & -\left(p_{4}-c_{k} \mu^{2}\right) & -p_{3}
\end{array}\right), \\
A_{k_{(3)}}=\left(\begin{array}{cccc}
0 & 0 & 0 & 0 \\
0 & 0 & -\mu^{2} c_{k} p_{2} & 0 \\
0 & 0 & 0 & 0 \\
0 & c_{k} \mu & 0 & 0
\end{array}\right) .
\end{gathered}
$$

$A_{k_{(3)}}$ is a nilpotent matrix with $\left(A_{k_{(3)}}\right)^{m}=\mathbf{0}$ for $m>2$, hence its exponential can be given exactly. For the matrices $A_{k_{(1)}}$ and $A_{k_{(2)}} A_{k_{(1)}} A_{k_{(2)}}=\mathbf{0}$ and $A_{k_{(2)}} A_{k_{(1)}}=\mathbf{0}$, so $\left[A_{k_{(1)}}, A_{k_{(2)}}\right]=\mathbf{0}$. On the one hand we win some adventage, due to the Statement 1 , and on the other hand, this decomposition has a disadvantage, namely we lost the property of exact solvability.

In the following decomposition, the original matrix $A_{k}$ is written as the sum of four matrices, two of them are commuting, and each has the property of exact solvability. The decomposition is the following:

$$
A_{k}=A_{k_{(1)}}+A_{k_{(2)}}+A_{k_{(3)}}+A_{k_{(4)}}
$$

where

$$
A_{k_{(1)}}=\left(\begin{array}{cccc}
0 & 0 & 0 & 0 \\
0 & -\left(p_{1}+p_{2} \mu c_{k}\right) & 0 & 0 \\
0 & 0 & 0 & 0 \\
0 & 0 & 0 & 0
\end{array}\right), \quad A_{k_{(2)}}=\left(\begin{array}{cccc}
0 & 0 & 0 & 0 \\
0 & 0 & 0 & 0 \\
0 & 0 & 0 & 0 \\
0 & 0 & 0 & -p_{3}
\end{array}\right),
$$




$$
A_{k_{(3)}}=\left(\begin{array}{cccc}
0 & 1 & 0 & 0 \\
0 & 0 & -\mu^{2} c_{k} p_{2} & 0 \\
0 & 0 & 0 & 1 \\
0 & 0 & 0 & 0
\end{array}\right), \quad A_{k_{(4)}}=\left(\begin{array}{cccc}
0 & 0 & 0 & 0 \\
-1 & 0 & 0 & 0 \\
0 & 0 & 0 & 0 \\
0 & c_{k} \mu & -\left(p_{4}-c_{k} \mu^{2}\right) & 0
\end{array}\right) .
$$

$A_{k_{(1)}}$ and $A_{k_{(2)}}$ are diagonal matrices (so they commute), therefore in both cases we can solve the subproblems exactly. Further, matrices $A_{k_{(3)}}$ and $A_{k_{(4)}}$ are nilpotent, so we can produce the exact solutions of the subproblems.

The last decomposition is the following:

$$
A_{k}=A_{k_{(1)}}+A_{k_{(2)}}+A_{k_{(3)}}+A_{k_{(4)}}
$$

where

$$
\begin{aligned}
A_{k_{(1)}}= & \left(\begin{array}{cccc}
0 & 1 & 0 & 0 \\
-1 & 0 & 0 & 0 \\
0 & 0 & 0 & 0 \\
0 & 0 & 0 & 0
\end{array}\right), \\
A_{k_{(3)}} & =\left(\begin{array}{cccc}
0 & 0 & 0 & 0 \\
0 & -\left(p_{1}+p_{2} \mu c_{k}\right) & 0 & 0 \\
0 & 0 & 0 & 0 \\
0 & 0 & 0 & -p_{3}
\end{array}\right), A_{k_{(2)}}=\left(\begin{array}{cccc}
0 & 0 & 0 & 0 \\
0 & 0 & 0 & 0 \\
0 & 0 & 0 & 1 \\
0 & 0 & -\left(p_{4}-c_{k} \mu^{2}\right) & 0
\end{array}\right),
\end{aligned}
$$

In this set matrices $A_{k_{(1)}}$ and $A_{k_{(2)}}$ commute, and the exponential of $A_{k_{(1)}}$ can be computed exactly (it is actually a rotation matrix). Matrices $A_{k_{(3)}}$ and $A_{k_{(4)}}$ are nilpotent and diagonal matrices, already discussed in previous decompositions.

\section{NumericAl ExPERIMENTS}

For the numerical implementation of operator splitting, the question is the following: what kind of methods to use for computing the solutions of subproblems? Here we considered three possibilities.

The first and perhaps the most obvious choice is when at every step we solve the subproblems by a numerical method with the same order of accuracy as that of the splitting method. This means that for sequential splitting we have to use a first-order method, such as the explicit or implicit Euler method. For Strang-Marchuk splitting and the Average Method we have to use a second-order method, e.g. second-order Euler or trapezoidal method.

The solution of the subproblems can also be given by truncating the infinite exponential sequence at the order of the splitting method. Finally, we can produce exact solutions by using decompositions of matrix $A_{k}$ to yield exactly solvable subproblems. We have already seen such decompositions in the previous section.

We performed the following eight experiments to solve the Cauchy problem (23): 
E1: Sequential splitting with decomposition (24), solving the subproblems corresponding to $A_{k_{(1)}}$ and $A_{k_{(2)}}$ with explicit Euler method and exactly, respectively.

E2: Sequential splitting with decomposition (25), all subproblems solved exactly.

E3: Explicit Euler solution of the full problem (no splitting).

E4: Strang-Marchuk splitting with decomposition (24), solving the subproblems corresponding to $A_{k_{(1)}}$ and $A_{k_{(2)}}$ with improved Euler method and exactly, respectively.

E5: Strang-Marchuk splitting with decomposition (25), all subproblems solved exactly.

E6: Average Method with sequential splitting and decomposition (24), solving the subproblems corresponding to $A_{k_{(1)}}$ and $A_{k_{(2)}}$ with explicit Euler method and exactly, respectively.

E7: Average Method with sequential splitting and decomposition (26) all subproblems solved with explicit Euler method in parallel.

E8: Improved Euler solution of the full problem (no splitting).

We now detail the results of the above numerical experiments. The parameter value $\mu=0.2$ and the initial condition $\mathbf{x}_{0}=(1,1,1,1)$ was used in all computations.

E1: Figure 1 shows component $x_{3}(t)$ of the solution on the time interval $[0,100]$. The figure shows how the splitting solution (red line) approximates the exact solution (blue line) by reducing the step size $h$.
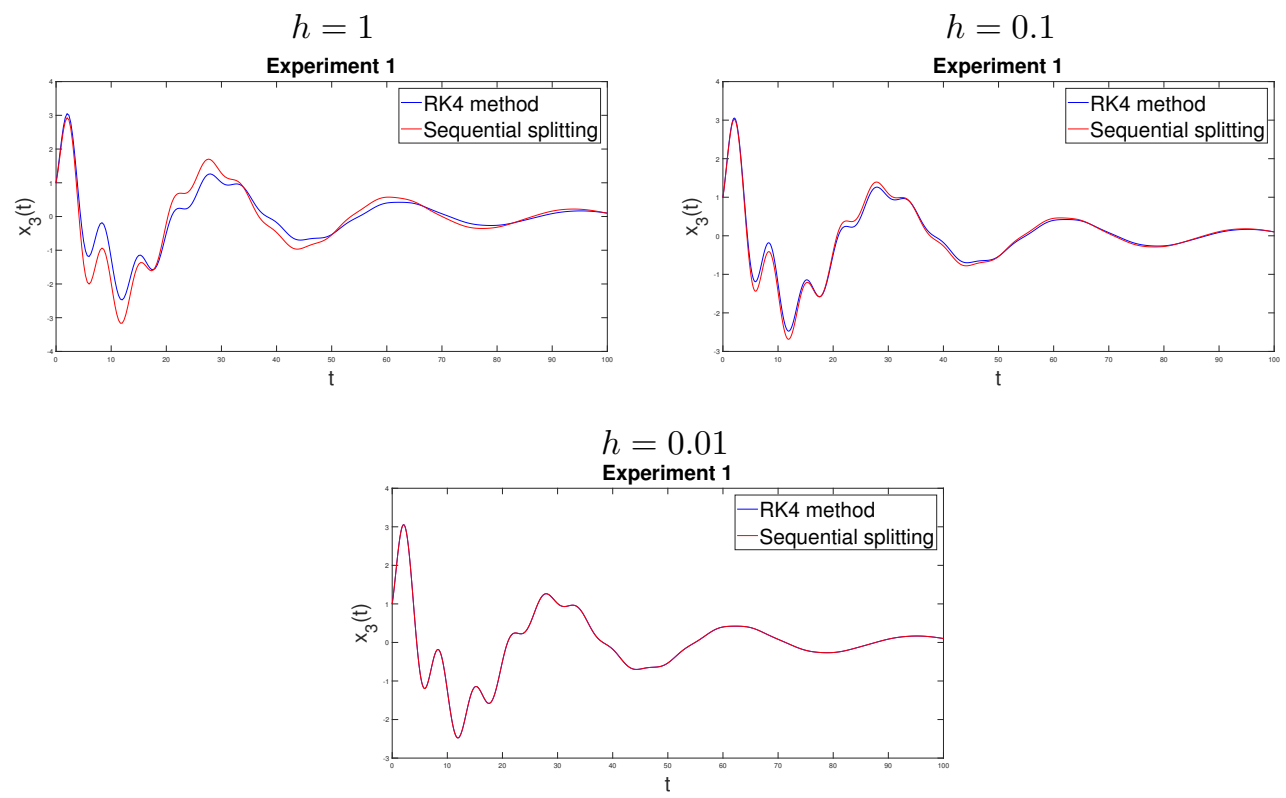

Figure 1. Experiment 1: $x_{3}(t)$ on time interval $[0,100]$ 

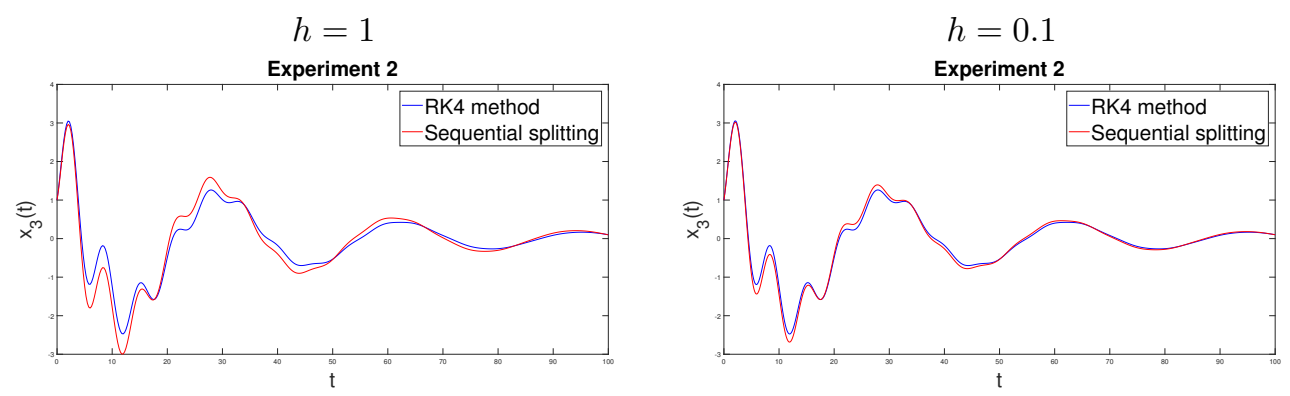

Figure 2. Experiment 2: $x_{3}(t)$ on the time interval $[0,100]$

E2: In Figure 2 we see how the splitting solution approximates the exact solution by reducing the step size $h$. Figure 2 shows component $x_{3}$ of the solution on the time interval $[0,100]$. The first 3 experiments utilized first-order methods. Runtimes are shown in Table 2 .

Table 2. Comparison of runtimes (in seconds) for E1-3

\begin{tabular}{|l||c||c||c|}
\hline $\mathbf{h}$ & $\mathbf{1 .}$ & $\mathbf{2 .}$ & $\mathbf{3 .}$ \\
\hline 1.0 & $7.02 \times 10^{-5}$ & $2.51 \times 10^{-5}$ & $2.39 \times 10^{-3}$ \\
\hline 0.1 & $8.44 \times 10^{-4}$ & $8.93 \times 10^{-4}$ & $5.32 \times 10^{-3}$ \\
\hline 0.01 & $1.70 \times 10^{-3}$ & $8.11 \times 10^{-3}$ & $1.09 \times 10^{-2}$ \\
\hline 0.001 & $1.37 \times 10^{-2}$ & $4.02 \times 10^{-2}$ & $7.14 \times 10^{-1}$ \\
\hline
\end{tabular}

As expected, we see that for the same order of accuracy splitting methods are faster than the full numerical solution. It can be seen that by reducing the step size $h$, the solvers containing splitting produce the numerical solution 1-2 orders of magnitude faster than the Euler method.

Table 3 shows the errors for Experiments 1 and 2. The methods give approximately the same error.

Table 3. Comparison of errors for Experiments 1 and 2

\begin{tabular}{|l||c||c|}
\hline $\mathbf{h}$ & \multicolumn{1}{c|}{$\mathbf{1 .}$} & $\mathbf{2 .}$ \\
\hline 1.0 & $2.56 \times 10^{-1}$ & $2.64 \times 10^{-1}$ \\
\hline 0.1 & $2.53 \times 10^{-2}$ & $2.09 \times 10^{-2}$ \\
\hline 0.01 & $2.09 \times 10^{-3}$ & $2.05 \times 10^{-3}$ \\
\hline 0.001 & $2.08 \times 10^{-4}$ & $2.04 \times 10^{-4}$ \\
\hline
\end{tabular}

E4: Since the Strang-Marchuk method is second-order, the choice of a bigger step size $h$ is also sufficient to obtain a well-approximating splitting solution. This is illustrated in Figure 3 , which shows $x_{4}(t)$ for the time interval $[0,100]$. We see that even for $h=0.3$ the splitting solution and the exact solution are very close. 
$h=1$

Experiment 4

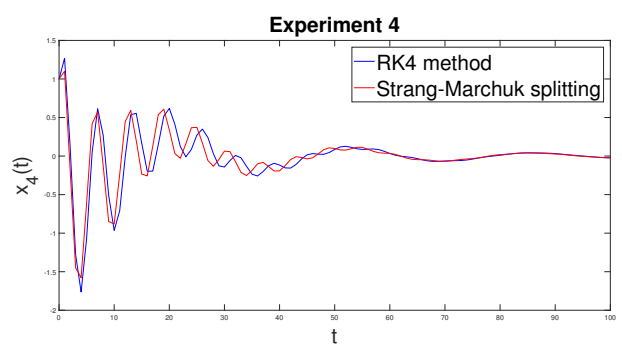

$h=0.1$

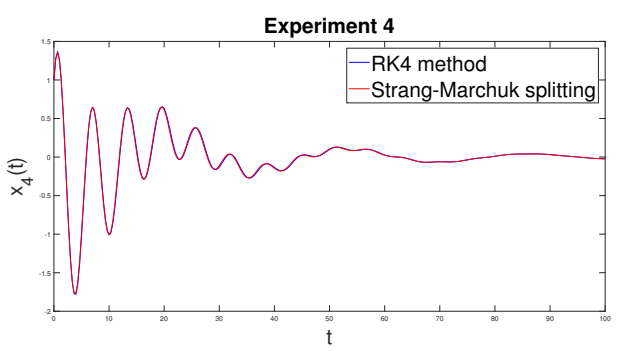

Figure 3. Experiment 4: $x_{4}(t)$ on the time interval $[0,100]$

E5: We see in Figure 4 how the splitting solution behaves when the step size $h$ is reduced. The figures show the component $x_{4}$ of the splitting solution on time interval $[0,100]$.

$h=1$

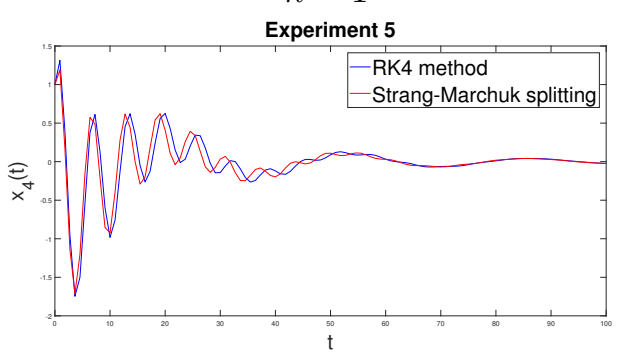

$h=0.1$

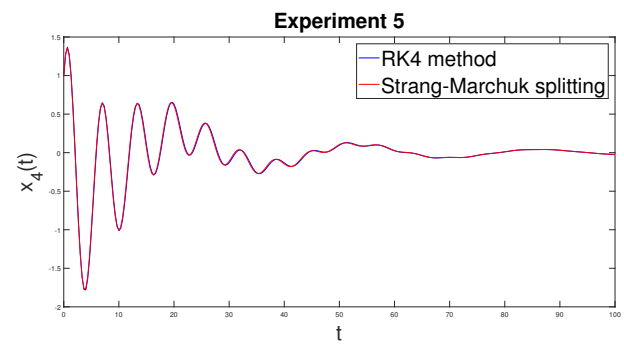

Figure 4. Experiment 5: $x_{4}(t)$ of the splitting solution on interval $[0,100]$

For E4 and E5 the errors as well as the runtimes are similar. It is interesting to compare the errors between the Strang-Marchuk splitting method and using a second-order numerical method without splitting. We used the second-order Euler method to solve the system (23) without splitting in Experiment 8. In Table 4 we show the errors. The errors are two orders of magnitude smaller for the same stepsize $h$ using the splitting method than using the second-order Euler method.

Table 4. Comparison of errors in case of Experiment 4 and 8

\begin{tabular}{|l||l||l|}
\hline h & Experiment 4 & Experiment 8 \\
\hline 1.0 & $7.20 \times 10^{-4}$ & $8.21 \times 10^{-2}$ \\
\hline 0.1 & $4.90 \times 10^{-7}$ & $8.24 \times 10^{-5}$ \\
\hline 0.01 & $4.78 \times 10^{-10}$ & $7.52 \times 10^{-8}$ \\
\hline 0.001 & $4.76 \times 10^{-13}$ & $7.43 \times 10^{-11}$ \\
\hline
\end{tabular}




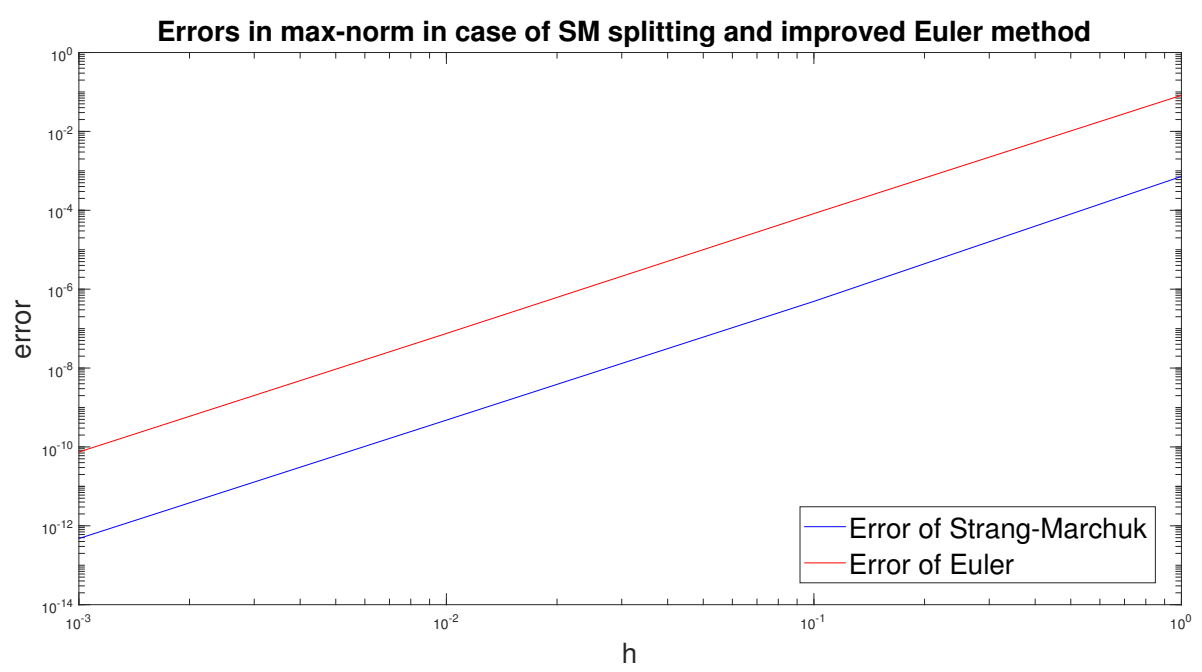

Figure 5. Error as a function of the stepsize $h$ in Experiments 4 and 8

Figure 5 shows how errors change with decreasing $h$ for Experiments 4 and 8 .

E6: In this experiment we apply the average sequential splitting. At first we use the decomposition 24 requiring 2 numerical solutions. For this case, we have to solve the following two subproblems at each step:

$$
\left\{\begin{array}{l}
\dot{y_{1}}(t)=A_{0_{(1)}} y_{1}(t) \\
y_{1}\left(t_{i}\right)=x_{s p(1)}\left(t_{i}\right),
\end{array} \quad t \in\left[t_{i}, t_{i+1}\right], \quad\left\{\begin{array}{l}
\dot{y_{2}}(t)=A_{0_{(2)}} y_{2}(t) \quad t \in\left[t_{i}, t_{i+1}\right] \\
y_{2}\left(t_{i}\right)=y_{1}\left(t_{i+1}\right) .
\end{array}\right.\right.
$$

The splitting solution at $t_{i+1}(i=0, \ldots, n-1)$ is

$$
\begin{aligned}
& x_{s p(1)}\left(t_{i+1}\right)= \\
& \quad=\exp \left(A_{0_{(2)}}\left(t_{i+1}-t_{i}\right)\right) y_{1}\left(t_{i+1}\right) \exp \left(A_{0_{(1)}}\left(t_{i+1}-t_{i}\right)\right) x_{s p(1)}\left(t_{i}\right) .
\end{aligned}
$$

We have to solve the following subproblems at each step:

$$
\left\{\begin{array}{l}
\dot{y}_{1}(t)=A_{0_{(2)}} y_{1}(t) \\
y_{1}\left(t_{i}\right)=x_{s p(2)}\left(t_{i}\right),
\end{array} \quad t \in\left[t_{i}, t_{i+1}\right], \quad\left\{\begin{array}{l}
\dot{y_{2}}(t)=A_{0_{(1)}} y_{2}(t) \quad t \in\left[t_{i}, t_{i+1}\right] \\
y_{2}\left(t_{i}\right)=y_{1}\left(t_{i+1}\right) .
\end{array}\right.\right.
$$

The form of the splitting solution at $t_{i+1}(i=0, \ldots, n-1)$ is

$$
\begin{aligned}
& x_{s p(2)}\left(t_{i+1}\right)= \\
& \quad=\exp \left(A_{0_{(1)}}\left(t_{i+1}-t_{i}\right)\right) y_{1}\left(t_{i+1}\right) \exp \left(A_{0_{(2)}}\left(t_{i+1}-t_{i}\right)\right) x_{s p(2)}\left(t_{i}\right) .
\end{aligned}
$$


The second-order approximate splitting solution is given by the average of the solutions $x_{s p(1)}\left(t_{i+1}\right)$ and $x_{s p(2)}\left(t_{i+1}\right)$, i.e.

$$
x_{s p}\left(t_{i+1}\right)=\frac{x_{s p(1)}\left(t_{i+1}\right)+x_{s p(2)}\left(t_{i+1}\right)}{2} .
$$

Figure 6 shows how the splitting solution approximates the exact solution with decreasing $h$. The figures show $x_{4}(t)$ on the $[0,100]$ time interval.
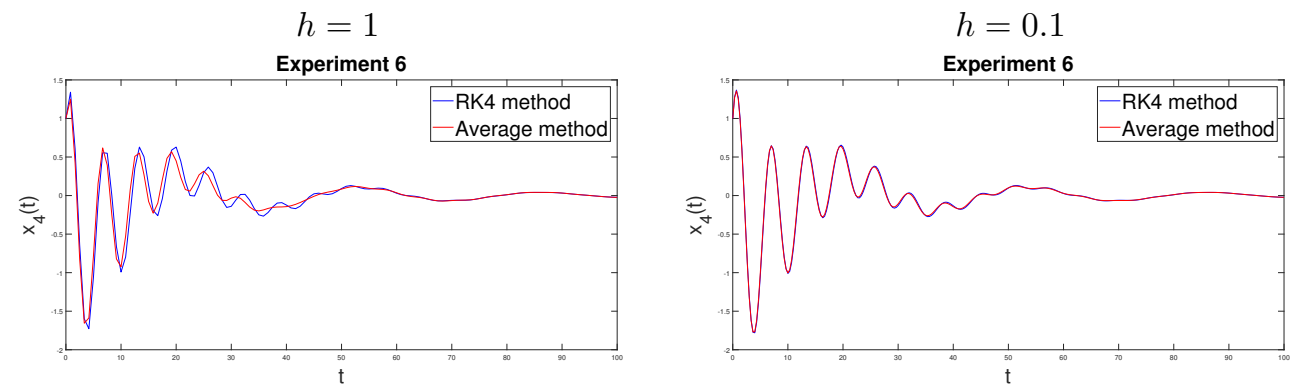

Figure 6. Experiment 6: $x_{4}(t)$ of the splitting solution on time interval $[0,100]$

E7: We consider the decomposition 26 where the first two matrices are commuting. Therefore, for using the Average Method instead of $3 !=6$ split problems we have to solve four subproblems, only.

The decomposition has the following form

$$
A_{0}=A_{0_{(3)}}+A_{0_{(4)}},
$$

where we use the notation $A_{0_{(4)}}$ for the sum of the two commuting matrices, i.e. $A_{0_{(4)}}:=A_{0_{(1)}}+A_{0_{(2)}}$, which means that

$$
A_{0_{(4)}}=\left(\begin{array}{cccc}
0 & 1 & 0 & 0 \\
-1 & -\left(p_{1}+p_{2} \mu c_{k}\right) & 0 & 0 \\
0 & 0 & 0 & 1 \\
0 & 0 & -\left(p_{4}-c_{k} \mu^{2}\right) & -p_{3}
\end{array}\right) .
$$

Thus, in these cases, we solve the following sequences of sub-problems.

First, we solve the following two subproblems where the commutativity is not present.

The ordering $A_{1} \rightarrow A_{3} \rightarrow A_{2}$ results in the following split sub-problems:

$$
\left\{\begin{array}{l}
\dot{y}_{1}(t)=A_{0_{(1)}} y_{1}(t) \\
y_{1}\left(t_{i}\right)=x_{s p(1)}\left(t_{i}\right),
\end{array} \quad t \in\left[t_{i}, t_{i+1}\right], \quad\left\{\begin{array}{l}
\dot{y_{2}}(t)=A_{0_{(3)}} y_{2}(t) \quad t \in\left[t_{i}, t_{i+1}\right] \\
y_{2}\left(t_{i}\right)=y_{1}\left(t_{i+1}\right),
\end{array}\right.\right.
$$




$$
\left\{\begin{array}{l}
\dot{y_{3}}(t)=A_{0_{(2)}} y_{3}(t) \quad t \in\left[t_{i}, t_{i+1}\right] \\
y_{3}\left(t_{i}\right)=y_{2}\left(t_{i+1}\right) .
\end{array}\right.
$$

Then the splitting solution at $t=t_{i+1}$ is

$$
\begin{aligned}
& x_{s p(1)}\left(t_{i+1}\right)= \\
= & \exp \left(A_{0_{(2)}}\left(t_{i+1}-t_{i}\right)\right) y_{2}\left(t_{i+1}\right) \exp \left(A_{0_{(3)}}\left(t_{i+1}-t_{i}\right)\right) y_{1}\left(t_{i+1}\right) \exp \left(A_{0_{(1)}}\left(t_{i+1}-t_{i}\right)\right) x_{s p(1)}\left(t_{i}\right) .
\end{aligned}
$$

The ordering $A_{2} \rightarrow A_{3} \rightarrow A_{1}$ results in the sub-problems following three subproblems:

$$
\begin{aligned}
& \left\{\begin{array}{l}
\dot{y}_{1}(t)=A_{0_{(2)}} y_{1}(t) \\
y_{1}\left(t_{i}\right)=x_{s p(2)}\left(t_{i}\right),
\end{array} \quad t \in\left[t_{i}, t_{i+1}\right], \quad\left\{\begin{array}{l}
\dot{y}_{2}(t)=A_{0_{(3)}} y_{2}(t) \quad t \in\left[t_{i}, t_{i+1}\right], \\
y_{2}\left(t_{i}\right)=y_{1}\left(t_{i+1}\right),
\end{array}\right.\right. \\
& \left\{\begin{array}{l}
\dot{y}_{3}(t)=A_{0_{(1)}} y_{1}(t) \quad t \in\left[t_{i}, t_{i+1}\right] \\
y_{3}\left(t_{i}\right)=y_{2}\left(t_{i+1}\right) .
\end{array}\right.
\end{aligned}
$$

Then the splitting solution at $t_{i+1}$ is

$$
\begin{aligned}
& x_{s p(2)}\left(t_{i+1}\right)= \\
= & \exp \left(A_{0_{(1)}}\left(t_{i+1}-t_{i}\right)\right) y_{2}\left(t_{i+1}\right) \exp \left(A_{0_{(3)}}\left(t_{i+1}-t_{i}\right)\right) y_{1}\left(t_{i+1}\right) \exp \left(A_{0_{(2)}}\left(t_{i+1}-t_{i}\right)\right) x_{s p(2)}\left(t_{i}\right) .
\end{aligned}
$$

Due to the commutativity, the remaining two problems, which we solve, consist of only two subproblems. These are the following.

For the ordering $A_{4} \rightarrow A_{3}$ the subproblems are the following:

$$
\left\{\begin{array}{l}
\dot{y_{1}}(t)=A_{0_{(4)}} y_{1}(t) \\
y_{1}\left(t_{i}\right)=x_{s p(3)}\left(t_{i}\right),
\end{array} \quad t \in\left[t_{i}, t_{i+1}\right], \quad\left\{\begin{array}{l}
\dot{y_{2}}(t)=A_{0_{(3)}} y_{2}(t) \quad t \in\left[t_{i}, t_{i+1}\right] \\
y_{2}\left(t_{i}\right)=y_{1}\left(t_{i+1}\right) .
\end{array}\right.\right.
$$

The splitting solution at $t_{i+1}$ is

$$
\begin{aligned}
x_{s p(3)}\left(t_{i+1}\right)= & \\
& =\exp \left(A_{0_{(3)}}\left(t_{i+1}-t_{i}\right)\right) y_{1}\left(t_{i+1}\right) \exp \left(A_{0_{(4)}}\left(t_{i+1}-t_{i}\right)\right) x_{s p(3)}\left(t_{i}\right) .
\end{aligned}
$$

Finally, for the ordering $A_{3} \rightarrow A_{4}$ we get

$$
\left\{\begin{array}{l}
\dot{y}_{1}(t)=A_{0_{(3)}} y_{1}(t) \\
y_{1}\left(t_{i}\right)=x_{s p(4)}\left(t_{i}\right),
\end{array} \quad t \in\left[t_{i}, t_{i+1}\right], \quad\left\{\begin{array}{l}
\dot{y}_{2}(t)=A_{0_{(4)}} y_{2}(t) \quad t \in\left[t_{i}, t_{i+1}\right] \\
y_{2}\left(t_{i}\right)=y_{1}\left(t_{i+1}\right) .
\end{array}\right.\right.
$$


Then

$$
\begin{aligned}
x_{s p(4)}\left(t_{i+1}\right)= & \\
& =\exp \left(A_{0_{(4)}}\left(t_{i+1}-t_{i}\right)\right) y_{1}\left(t_{i+1}\right) \exp \left(A_{0_{(3)}}\left(t_{i+1}-t_{i}\right)\right) x_{s p(4)}\left(t_{i}\right) .
\end{aligned}
$$

Using the Average Method, the second-order accurate approximation is defined as

$$
x_{s p}(t)=\frac{x_{s p(1)}(t)+x_{s p(2)}(t)+2 \cdot x_{s p(3)}(t)+2 \cdot x_{s p(4)}(t)}{6} .
$$

Figure 7 shows how the 4 th component $\left(x_{4}(t)\right)$ of the splitting solution approaches the exact solution with decreasing $h$. The advantage of the method is
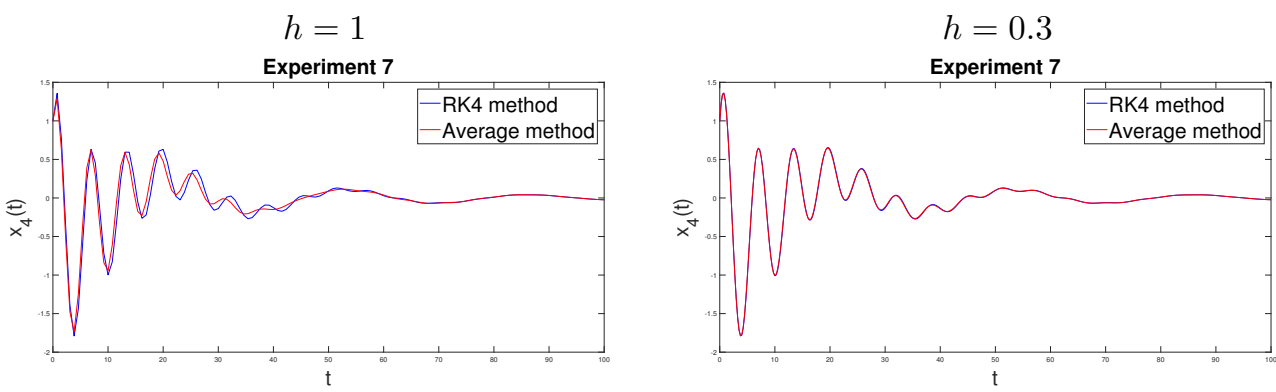

Figure 7. Experiment 7: $x_{4}(t)$ on time interval interval $[0,100]$

that the solutions (40), (44), (47) and $(50)$ can be independently calculated, i.e., the computation is parallelizable.

Table 5. Comparison of runtimes (in seconds) for Experiments 4-8.

\begin{tabular}{|l||l|l||l|c||l|}
\hline $\mathbf{h}$ & \multicolumn{1}{|c|}{$\mathbf{E 4}$} & \multicolumn{1}{|c|}{ E5 } & E6 & E7 & E8 \\
\hline 1.0 & $4.32 \times 10^{-2}$ & $7.57 \times 10^{-2}$ & $1.15 \times 10^{-4}$ & $4.54 \times 10^{-4}$ & $8.18 \times 10^{-3}$ \\
\hline 0.1 & $7.55 \times 10^{-1}$ & $8.71 \times 10^{-1}$ & $1.01 \times 10^{-3}$ & $1.52 \times 10^{-3}$ & $1.96 \times 10^{-2}$ \\
\hline 0.01 & $5.20 \times 10^{0}$ & $6.39 \times 10^{0}$ & $3.65 \times 10^{-3}$ & $7.81 \times 10^{-3}$ & $8.44 \times 10^{-2}$ \\
\hline 0.001 & $1.53 \times 10^{1}$ & $2.06 \times 10^{1}$ & $1.89 \times 10^{-2}$ & $6.47 \times 10^{-2}$ & $1.13 \times 10^{0}$ \\
\hline
\end{tabular}

Table 5 collects the comparison of runtimes for Experiments 4-8. These results show that sequential and Strang-Marchuk splitting is about two orders of magnitude slower than the improved Euler method, while the Average Method is about two orders of magnitude faster. This is an encouraging result for the applicability of the Average Method. 


\section{SUMMARY}

By performing several numerical experiments we demonstrated that the benefits of the Average Method are the following:

- easy implementation when $d$ is small,

- provides a second-order approximation solution using a first-order method,

- the numerical solutions of the subproblems can be independently computed, therefore the method can be parallelized.

The drawback of the Average Method is the large number $(d !)$ of solutions to be computed. This number can be reduced by finding a decomposition of the system matrix into a set of pairwise commuting matrices.

Acknowledgements. This work was completed in the ELTE Institutional Excellence Program (TKP2020-IKA-05) financed by the Hungarian Ministry of Human Capacities, Istvan Farago has been supported by the European Union, and co-financed by the European Social Fund (EFOP-3.6.3-VEKOP-16-2017-00002) and was also supported by the Hungarian Scientific Research Fund OTKA, SNN125119. The work of T. Kalmár-Nagy is supported by the NRDI Funds (TKP2020 IES, Grant No. BME-IE-WAT; TKP2020 NC, Grant No. BME-NC) based on the charter of bolster issued by the NRDI Office under the auspices of the Ministry for Innovation and Technology.

\section{APPENDIX 1}

Statement 1. Assume that we have the Cauchy problem (1) Solving this problem in all possible sequences using sequential splitting, and then taking the average of the resulting numerical solutions, the method is second-order, i.e.

$$
\exp \left(h\left(A_{1}+\ldots+A_{d}\right)\right)=\frac{1}{d !} \sum_{p \in \mathcal{P}^{d}} \exp \left\{p_{1}, p_{2}, \ldots, p_{n}\right\}+\mathcal{O}\left(h^{3}\right) .
$$

Proof. We prove the statement by induction. First we consider the case $k=2$, i.e. the validity of the formula

$$
\exp \left(h\left(A_{1}+A_{2}\right)\right)=\frac{\exp \left(h A_{1}\right) \exp \left(h A_{2}\right)+\exp \left(h A_{2}\right) \exp \left(h A_{1}\right)}{2 !}+\mathcal{O}\left(h^{3}\right) .
$$

Obviously, for any matrix $C$ we have

$$
\exp (h C)=I+h C+\frac{h^{2}}{2} C^{2}+\mathcal{O}\left(h^{3}\right)
$$

Hence,

$$
\begin{gathered}
\frac{\exp \left(h A_{1}\right) \exp \left(h A_{2}\right)+\exp \left(h A_{2}\right) \exp \left(h A_{1}\right)}{2 !}= \\
=\frac{\left[I+h A_{1}+\frac{h^{2}}{2 !} A_{1}^{2}\right]\left[I+h A_{2}+\frac{h^{2}}{2 !} A_{2}^{2}\right]+\left[I+h A_{2}+\frac{h^{2}}{2 !} A_{2}^{2}\right]\left[I+h A_{1}+\frac{h^{2}}{2 !} A_{1}^{2}\right]}{2 !}= \\
=\frac{2 I+2 h\left(A_{1}+A_{2}\right)+h^{2}\left(A_{1}^{2}+A_{2}^{2}+A_{1} A_{2}+A_{2} A_{1}\right)}{2 !}= \\
=I+h\left(A_{1}+A_{2}\right)+\frac{h^{2}}{2 !}\left(A_{1}^{2}+A_{2}^{2}+A_{1} A_{2}+A_{2} A_{1}\right)
\end{gathered}
$$


which proves 52 .

Now we suppose that the statement is true for $k=d$ and prove its validity for $k=d+1$.

$$
\begin{aligned}
& \exp \left(h\left(A_{1}+A_{2}+\ldots+A_{d}+A_{d+1}\right)\right)= \\
& =I+h \sum_{j=1}^{d+1} A_{j}+\frac{h^{2}}{2 !}\left(\sum_{j=1}^{d+1} A_{j}^{2}+\sum_{i=1}^{d} \sum_{j=i+1}^{d+1} A_{i} A_{j}+\sum_{i=2}^{d+1} \sum_{j=1}^{i-1} A_{i} A_{j}\right)+\mathcal{O}\left(h^{3}\right) .
\end{aligned}
$$

Using the notation $B=A_{1}+A_{2}+\ldots+A_{d}$, we have

$$
\begin{aligned}
& \exp \left(h\left(A_{1}+A_{2}+\ldots+A_{d}+A_{d+1}\right)\right)= \\
& =\exp \left(h\left(B+A_{d+1}\right)\right)=\frac{\exp (h B) \exp \left(h A_{d+1}\right)+\exp \left(h A_{d+1}\right) \exp (h B)}{2 !}+\mathcal{O}\left(h^{3}\right)= \\
& =\frac{1}{2}\left[I+h \sum_{j=1}^{d} A_{j}+\frac{h^{2}}{2 !}\left(\sum_{j=1}^{d} A_{j}^{2}+\sum_{i=1}^{d-1} \sum_{j=i+1}^{d} A_{i} A_{j}+\sum_{i=2}^{d} \sum_{j=1}^{i-1} A_{i} A_{j}\right)+\right. \\
& \left.+h A_{d+1}+h^{2} \sum_{j=1}^{d} A_{j} A_{d+1}+\frac{h^{2}}{2} A_{d+1}^{2}\right]+ \\
& +\frac{1}{2}\left[I+h \sum_{j=1}^{d} A_{j}+\frac{h^{2}}{2 !}\left(\sum_{j=1}^{d} A_{j}^{2}+\sum_{i=1}^{d-1} \sum_{j=i+1}^{d} A_{i} A_{j}+\sum_{i=2}^{d} \sum_{j=1}^{i-1} A_{i} A_{j}\right)+\right. \\
& \left.+h A_{d+1}+h^{2} \sum_{j=1}^{d} A_{d+1} A_{j}+\frac{h^{2}}{2} A_{d+1}^{2}\right]+\mathcal{O}\left(h^{3}\right)= \\
& =\frac{1}{2}\left[2 I+2 h\left(\sum_{j=1}^{d} A_{j}+A_{d+1}\right)+h^{2}\left(\sum_{j=1}^{d} A_{j}^{2}+A_{d+1}^{2}+\sum_{i=1}^{d-1} \sum_{j=i+1}^{d} A_{i} A_{j}+\right.\right. \\
& \left.\left.+\sum_{i=2}^{d} \sum_{j=1}^{i-1} A_{i} A_{j}+\sum_{j=1}^{d} A_{d+1} A_{j}+A_{j} A_{d+1}\right)\right]+\mathcal{O}\left(h^{3}\right)= \\
& =\frac{1}{2}\left[2 I+2 h \sum_{j=1}^{d+1} A_{j}+h^{2}\left(\sum_{j=1}^{d+1} A_{j}^{2}+\sum_{i=1}^{d} \sum_{j=i+1}^{d+1} A_{i} A_{j}+\right.\right. \\
& \left.\left.+\sum_{i=2}^{d+1} \sum_{j=1}^{i-1} A_{i} A_{j}\right)\right]+\mathcal{O}\left(h^{3}\right)= \\
& =I+h \sum_{j=1}^{d+1} A_{j}+\frac{h^{2}}{2}\left(\sum_{j=1}^{d+1} A_{j}^{2}+\sum_{i=1}^{d} \sum_{j=i+1}^{d+1} A_{i} A_{j}+\right. \\
& \left.+\sum_{i=2}^{d+1} \sum_{j=1}^{i-1} A_{i} A_{j}\right)+\mathcal{O}\left(h^{3}\right)
\end{aligned}
$$

This proves our statement. 
APPENDIX 2

Statement 2. If and only if $A=A_{1}+A_{2}$, and $A_{1}$ and $A_{2}$ satisfy the condition $\left[A_{1},\left[A_{1}, A_{2}\right]\right]=\left[A_{2},\left[A_{1}, A_{2}\right]\right]$ then

$$
\exp \left(h\left(A_{1}+A_{2}\right)\right)=\frac{\exp \left(h A_{1}\right) \exp \left(h A_{2}\right)+\exp \left(h A_{2}\right) \exp \left(h A_{1}\right)}{2}+\mathcal{O}\left(h^{4}\right)
$$

Proof. Let $x, y \in \mathbb{R} \backslash\{0\}, x+y \neq 0$ and $d=2$ and we want to prove the following relation:

$$
\exp \left(h\left(A_{1}+A_{2}\right)\right)=\frac{x \exp \left(h A_{1}\right) \exp \left(h A_{2}\right)+y \exp \left(h A_{2}\right) \exp \left(h A_{1}\right)}{x+y}+\mathcal{O}\left(h^{4}\right) .
$$

Clearly

$$
\begin{aligned}
\exp ( & \left.h\left(A_{1}+A_{2}\right)\right)= \\
=I & +h\left(A_{1}+A_{2}\right)+\frac{h^{2}}{2 !}\left(A_{1}+A_{2}\right)^{2}+\frac{h^{3}}{3 !}\left(A_{1}+A_{2}\right)^{3}+\mathcal{O}\left(h^{4}\right)= \\
=I & +h\left(A_{1}+A_{2}\right)+\frac{h^{2}}{2}\left(A_{1}^{2}+A_{2}^{2}+A_{1} A_{2}+A_{2} A_{1}\right)+\frac{h^{3}}{6}\left(A_{1}^{3}+A_{2}^{3}+\right. \\
& \left.\quad+A_{1} A_{2}^{2}+A_{1}^{2} A_{2}+A_{2} A_{1}^{2}+A_{2}^{2} A_{1}+A_{1} A_{2} A_{1}+A_{2} A_{1} A_{2}\right)+\mathcal{O}\left(h^{4}\right) .
\end{aligned}
$$

Then, the right-hand side of (57):

$$
\begin{aligned}
& \frac{x \exp \left(h A_{1}\right) \exp \left(h A_{2}\right)+y \exp \left(h A_{2}\right) \exp \left(h A_{1}\right)+\mathcal{O}\left(h^{4}\right)=}{x+y}= \\
& =\frac{1}{x+y}\left[x\left(I+h A_{1}+\frac{h^{2}}{2 !} A_{1}^{2}+\frac{h^{3}}{3 !} A_{1}^{3}+\mathcal{O}\left(h^{4}\right)\right)\left(I+h A_{2}+\frac{h^{2}}{2 !} A_{2}^{2}+\frac{h^{3}}{3 !} A_{2}^{3}+\mathcal{O}\left(h^{4}\right)\right)\right]+ \\
& +\frac{1}{x+y}\left[y\left(I+h A_{2}+\frac{h^{2}}{2 !} A_{2}^{2}+\frac{h^{3}}{3 !} A_{2}^{3}+\mathcal{O}\left(h^{4}\right)\right)\left(I+h A_{1}+\frac{h^{2}}{2 !} A_{1}^{2}+\frac{h^{3}}{3 !} A_{1}^{3}+\mathcal{O}\left(h^{4}\right)\right)\right]= \\
& =\frac{1}{x+y}\left[x \left(I+h\left(A_{1}+A_{2}\right)+h^{2}\left(\frac{1}{2} A_{1}^{2}+\frac{1}{2} A_{2}^{2}+A_{1} A_{2}\right)+\right.\right. \\
& \left.\left.+\quad+h^{3}\left(\frac{1}{6} A_{1}^{3}+\frac{1}{6} A_{2}^{3}+\frac{1}{2} A_{1}^{2} A_{2}+\frac{1}{2} A_{1} A_{2}^{2}\right)\right)\right]+ \\
& +\frac{1}{x+y}\left[y \left(I+h\left(A_{1}+A_{2}\right)+h^{2}\left(\frac{1}{2} A_{1}^{2}+\frac{1}{2} A_{2}^{2}+A_{2} A_{1}\right)+\right.\right. \\
& \left.\left.+h^{3}\left(\frac{1}{6} A_{1}^{3}+\frac{1}{6} A_{2}^{3}+\frac{1}{2} A_{2}^{2} A_{1}+\frac{1}{2} A_{2} A_{1}^{2}\right)\right)\right]= \\
& =I+h\left(A_{1}+A_{2}\right)+\frac{1}{x+y}\left\{h^{2}\left[x\left(\frac{1}{2} A_{1}^{2}+\frac{1}{2} A_{2}^{2}+A_{1} A_{2}\right)+y\left(\frac{1}{2} A_{1}^{2}+\frac{1}{2} A_{2}^{2}+A_{2} A_{1}\right)\right]+\right. \\
& \left.+h^{3}\left[x\left(\frac{1}{6} A_{1}^{3}+\frac{1}{6} A_{2}^{3}+\frac{1}{2} A_{1}^{2} A_{2}+\frac{1}{2} A_{1} A_{2}^{2}\right)+y\left(\frac{1}{6} A_{1}^{3}+\frac{1}{6} A_{2}^{3}+\frac{1}{2} A_{2}^{2} A_{1}+\frac{1}{2} A_{2} A_{1}^{2}\right)\right]\right\}
\end{aligned}
$$


57) will be true if coefficients of (58) and (59) are the same. The coefficients of $h^{i}$, $i=0,1,2,3$ are the following.

Coefficient of $h^{0}$ :

$$
\frac{(x+y) I}{(x+y)}=I \Longrightarrow I=I
$$

Coefficient of $h^{1}$ :

$$
\frac{(x+y)\left(A_{1}+A_{2}\right)}{(x+y)}=\left(A_{1}+A_{2}\right) \Longrightarrow\left(A_{1}+A_{2}\right)=\left(A_{1}+A_{2}\right) .
$$

Coefficient of $h^{2}$ :

$$
\begin{aligned}
x\left(\frac{1}{2} A_{1}^{2}+\frac{1}{2} A_{2}^{2}+A_{1} A_{2}\right)+y\left(\frac{1}{2} A_{1}^{2}+\frac{1}{2} A_{2}^{2}+A_{2} A_{1}\right)= & \\
& =\frac{1}{2}\left(A_{1}+A_{2}\right)+\frac{1}{2}\left(A_{1} A_{2}+A_{2} A_{1}\right) \\
\frac{1}{2}\left(A_{1}+A_{2}\right)+\frac{x A_{1} A_{2}+y A_{2} A_{1}}{x+y} & =\frac{1}{2}\left(A_{1}+A_{2}\right)+\frac{1}{2}\left(A_{1} A_{2}+A_{2} A_{1}\right), \\
\frac{x A_{1} A_{2}+y A_{2} A_{1}}{x+y} & =\frac{1}{2}\left(A_{1} A_{2}+A_{2} A_{1}\right), \\
x A_{1} A_{2}+y A_{2} A_{1} & =\frac{1}{2}(x+y) A_{1} A_{2}+\frac{1}{2}(x+y) A_{2} A_{1} . \\
\Downarrow & \\
x=y & =\frac{1}{2}(x+y), \\
x & =y .
\end{aligned}
$$

Coefficient of $h^{3}$ :

$$
\begin{array}{r}
\frac{1}{x+y}\left[x\left(\frac{1}{6} A_{1}^{3}+\frac{1}{6} A_{2}^{3}+\frac{1}{2} A_{1}^{2} A_{2}+\frac{1}{2} A_{1} A_{2}^{2}\right)+y\left(\frac{1}{6} A_{1}^{3}+\frac{1}{6} A_{2}^{3}+\frac{1}{2} A_{2}^{2} A_{1}+\frac{1}{2} A_{2} A_{1}^{2}\right)\right]= \\
=\frac{1}{6}\left(A_{1}^{3}+A_{2}^{3}+A_{1} A_{2}^{2}+A_{1}^{2} A_{2}+A_{2} A_{1}^{2}+A_{2}^{2} A_{1}+A_{1} A_{2} A_{1}+A_{2} A_{1} A_{2}\right) .
\end{array}
$$

After some calculations we get

$$
\begin{aligned}
& \frac{x\left(A_{1}^{2} A_{2}+A_{1} A_{2}^{2}\right)+y\left(A_{2}^{2} A_{1}+A_{2} A_{1}^{2}\right)}{2(x+y)}= \\
& \quad=\frac{A_{1} A_{2}^{2}+A_{1}^{2} A_{2}+A_{2} A_{1}^{2}+A_{2}^{2} A_{1}+A_{1} A_{2} A_{1}+A_{2} A_{1} A_{2}}{6},
\end{aligned}
$$

Utilizing equation (60) yields

$$
\begin{aligned}
\frac{x\left(A_{1}^{2} A_{2}+A_{1} A_{2}^{2}+A_{2}^{2} A_{1}+A_{2} A_{1}^{2}\right)}{4 x}= & \\
= & \frac{A_{1} A_{2}^{2}+A_{1}^{2} A_{2}+A_{2} A_{1}^{2}+A_{2}^{2} A_{1}+A_{1} A_{2} A_{1}+A_{2} A_{1} A_{2}}{6}
\end{aligned}
$$


or

and

$$
A_{1}^{2} A_{2}+A_{1} A_{2}^{2}+A_{2}^{2} A_{1}+A_{2} A_{1}^{2}=2 A_{1} A_{2} A_{1}+2 A_{2} A_{1} A_{2}
$$

$$
A_{1}\left[A_{1}, A_{2}\right]+\left[A_{2}, A_{1}\right] A_{1}+A_{2}\left[A_{2}, A_{1}\right]+\left[A_{1}, A_{2}\right] A_{2}=0 .
$$

On the basis of the well known equality $[A, B]=-[B, A]$ we have

$$
A_{1}\left[A_{1}, A_{2}\right]-\left[A_{1}, A_{2}\right] A_{1}-A_{2}\left[A_{1}, A_{2}\right]+\left[A_{1}, A_{2}\right] A_{2}=0,
$$

or

$$
\left[A_{1},\left[A_{1}, A_{2}\right]\right]+\left[\left[A_{1}, A_{2}\right], A_{2}\right]=0,
$$

and

$$
\left[A_{1},\left[A_{1}, A_{2}\right]\right]-\left[A_{2},\left[A_{1}, A_{2}\right]\right]=0 .
$$

Hence, the condition of third order is as follows

$$
\left[A_{1},\left[A_{1}, A_{2}\right]\right]=\left[A_{2},\left[A_{1}, A_{2}\right]\right] \text {. }
$$

This means that for a decomposition of two matrices the method will be third-order if and only if condition (61) is satisfied.

\section{REFERENCES}

1. R. I. McLachlan and G. Reinout W. Quispel. "Splitting methods." Acta Numerica, 11, (2002), pp. 341-434. DOI: 10.1017/S0962492902000053.

2. István Faragó and Ágnes Havasy. Operator splittings and their applications. Nova Science Publ., 2009.

3. Bei Gu and H. Harry Asada. "Co-Simulation of Algebraically Coupled Dynamic Subsystems Without Disclosure of Proprietary Subsystem Models." Journal of Dynamic Systems, Measurement, and Control, 126(1) (2004), pp. 1-13. DOI: 10. 1115/1.1648307.

4. Tamás Kalmár-Nagy and Ilinca Stanciulescu. "Can complex systems really be simulated?" Applied Mathematics and Computation, 227, (2014), pp. 199-211.

5. Petra Csomós and Gregor Nickel. "Operator splitting for delay equations." Computers \& Mathematics with Applications, 55(10), (2008), pp. 2234-2246. DOI: 10.1016/j.camwa.2007.11.011.

6. Kevin O'Meara, John Clark, and Charles Vinsonhaler. Advanced Topics in Linear Algebra: Weaving Matrix Problems through the Weyr Form. Oxford University Press, 2011.

7. Tamás Kalmár-Nagy, Rudolf Csikja, and Tarek A Elgohary. "Nonlinear analysis of a 2-DOF piecewise linear aeroelastic system." Nonlinear Dynamics, 85,(2) (2016), pp. 739-750. DOI: 10.1007/s11071-016-2719-z.

8. János Lelkes and Tamás Kalmár-Nagy. "Analysis of a piecewise linear aeroelastic system with and without tuned vibration absorber." Nonlinear Dynamics, (2020), pp. 1-22. DOI: $10.1007 / \mathrm{s} 11071-016-2719-\mathrm{z}$. 